\title{
Ramanujan's class invariants and cubic continued fraction
}

\author{
by
}

Bruce C. Berndt (Urbana, Ill.), Heng Huat Chan (Princeton, N.J.) and Liang-Cheng Zhang (Springfield, Mo.)

1. Introduction. To describe the purpose and content of this paper, we first need to define a modular equation and singular modulus before defining the two classical class invariants.

Let $K, K^{\prime}, L$, and $L^{\prime}$ denote complete elliptic integrals of the first kind associated with the moduli $k, k^{\prime}:=\sqrt{1-k^{2}}, l$, and $l^{\prime}:=\sqrt{1-l^{2}}$, respectively, where $0<k, l<1$. Suppose that

$$
\frac{K^{\prime}}{K}=n \frac{L^{\prime}}{L}
$$

for some positive rational number $n$. If $n$ is a positive integer, a relation between $k$ and $l$ induced by (1.1) is called a modular equation of degree $n$. As usual, in the theory of elliptic functions, set

$$
q:=\exp \left(-\pi K^{\prime} / K\right) .
$$

Suppose now that $k=l^{\prime}$, so that $k^{\prime}=l, K=L^{\prime}$, and $K^{\prime}=L$. It follows from (1.1) and (1.2) that

$$
q=\exp (-\pi \sqrt{n}) .
$$

The corresponding modulus $k=: k_{n}$ is called a singular modulus. Usually, $n$ is a positive integer, but this restriction is not necessary.

Following Ramanujan, set

$$
\alpha=k^{2} \text { and } \beta=l^{2} .
$$

Let

$$
(a ; q)_{\infty}=\prod_{n=0}^{\infty}\left(1-a q^{n}\right), \quad|q|<1 .
$$

Again, following Ramanujan [1, p. 37], we set

$$
\chi(q)=\left(-q ; q^{2}\right)_{\infty}
$$


Then [1, p. 124]

$$
\chi(q)=2^{1 / 6}\{\alpha(1-\alpha) / q\}^{-1 / 24}
$$

and

$$
\chi(-q)=2^{1 / 6}(1-\alpha)^{1 / 12}(\alpha / q)^{-1 / 24} .
$$

For $q=\exp (-\pi \sqrt{n})$, define the two class invariants $G_{n}$ and $g_{n}$ by

$$
G_{n}:=2^{-1 / 4} q^{-1 / 24} \chi(q) \text { and } g_{n}:=2^{-1 / 4} q^{-1 / 24} \chi(-q) \text {. }
$$

Since modular equations are algebraic equations in $\alpha$ and $\beta$, it follows from (1.5)-(1.7) that $G_{n}$ and $g_{n}$ are algebraic. As G. N. Watson [17] remarked, "For reasons which had commended themselves to Weber and Ramanujan independently, it is customary to determine $G_{n}$ for odd values of $n$, and $g_{n}$ for even values of $n$."

Weber [23] uses the notations $f(\sqrt{-n}):=2^{1 / 4} G_{n}$ and $f_{1}(\sqrt{-n}):=$ $2^{1 / 4} g_{n}$. The notation (1.7) is that used by Ramanujan in his famous paper [11], [13, pp. 23-39]. Since Watson employed this notation in his series of papers [16]-[22] on singular moduli, and since J. M. and P. B. Borwein also adhere to this notation in their recent treatise [4], we do so as well. However, it must be emphasized that Ramanujan's definition of $G_{n}$ in [11] is not the same as that used by him in his notebooks [12], while his definition of $g_{n}$ in [11] is that used in his first notebook but not in his second notebook.

Ramanujan calculated a prodigious number of class invariants. On pages 294-299 in his second notebook [12], Ramanujan listed a total of 77 class invariants. Most of these and several others are scattered throughout his first notebook [12]. His paper [11] contains 21 class invariants found in his second notebook and 25 further class invariants not given in his second notebook. Although Ramanujan [12] calculated several class invariants that were previously determined by Weber [23], the list in Ramanujan's paper has no intersection with Weber's table. In two papers [17], [18], Watson proved 24 class invariants found in Ramanujan's paper [11], but not recorded in his second notebook. However, except for $n=325$ and 363, the remaining 22 invariants were recorded by Ramanujan at scattered places in the first notebook. In his first paper [17], Watson employed an "empirical process" to establish 14 of the invariants. This process was also used by Watson [16] in proving the formula for $G_{1353}$, found in [11] and communicated by Ramanujan [13, p. xxix] in his second letter to G. H. Hardy. In the second paper [18], Watson employed modular equations to establish the remaining ten invariants found in Ramanujan's paper [11], but not in his second notebook. In the introduction to [17], Watson remarks "It is intended to publish the calculations involved in the construction of the set $N+Q$ (the invariants appearing in both Ramanujan's paper [11] and the second notebook) as part of the commentary on the note-books by Dr. B. M. Wilson and myself." 
Although Watson and Wilson's efforts to edit Ramanujan's notebooks have been preserved in the library at Trinity College, Cambridge, Watson's work on these 21 invariants is not found there. If Watson actually calculated these invariants, it appears that his work has been lost. The 21 values of $n$ are: $65,69,77,81,117,141,145,147,153,205,213,217,265,289,301,441,445$, 505, 553, 90, and 198.

After establishing 25 of Ramanujan's invariants in [16]-[18], Watson calculated several other class invariants in four further papers [19]-[22]. The values of $n$ considered by Watson depend upon the class numbers for positive definite quadratic forms of discriminant $-n$. In the course of his evaluations, he determined the class invariants for $n=81$ [21], 147 [21], and 289 [22]. Thus, 18 class invariants from the list of 21 have heretofore not been proved.

In this paper, we shall establish Ramanujan's class invariants for $n=117,153,441,90$, and 198. Note that for each such $n, 9 \mid n$. In another paper [3], we shall prove the remaining 13 class invariants. Our starting point is a relation connecting $g_{n}$ and $g_{9 n}$, found on page 318 of Ramanujan's first notebook, but not in his second notebook. K. G. Ramanathan [9], [10] noticed this relation in the first notebook, but apparently he never gave a proof. Also unaware of its appearance in the first notebook, J. M. and P. B. Borwein [4, pp. 145, 149], although not stating the results explicitly, derived a formula connecting $g_{n}$ and $g_{9 n}$, as well as a formula relating $G_{n}$ and $G_{9 n}$. They [4, pp. 145, 146] also derive formulas connecting $G_{81 n}$ with $G_{n}$ and $G_{9 n}$, and $g_{81 n}$ with $g_{n}$ and $g_{9 n}$. In the next section, we use one of Ramanujan's modular equations of degree 3 to establish the aforementioned formulas connecting $G_{n}$ and $G_{9 n}$, and $g_{n}$ and $g_{9 n}$. The former formula is not found in the notebooks, but it can be proved along the same lines as the latter.

The algebraic nature of class invariants depends upon properties such as the class number of $\mathbb{Q}(\sqrt{-n})$, the genus structure, the number of classes per genus, and the genus characters [3]. These features produce differences in each calculation. Thus, we were unable to shorten either of the two methods of verification used in the sequel by appealing to a set formula or procedure. Failing to obviate these difficulties, we were therefore also unable to mechanize the calculations.

Of course, the ideas in this paper can be utilized to establish other class invariants found by Ramanujan when $9 \mid n$, in particular, for $n=27$, $45,63,81,225,333,765,18,126,522$, and 630 . Undoubtedly, some of these proofs would be simpler than previous proofs, for example, for 81, 225, 333, $765,126,522$, and 630 . In particular, Watson's proofs for $n=333,765,522$, and 630 [17] were based on an "empirical method." In fact, the Borweins [4, pp. 147, 149, 150] employed the aforementioned formulas to calculate the invariants $G_{27}, G_{81}, G_{225}$, and $g_{522}$. 
Ramanujan's cubic continued fraction $G(q)$, first introduced by him in his second letter to Hardy [13, p. xxvii], is defined for $|q|<1$ by

$$
G(q):=\frac{q^{1 / 3}}{1}+\frac{q+q^{2}}{1}+\frac{q^{2}+q^{4}}{1}+\frac{q^{3}+q^{6}}{1}+\cdots .
$$

Chan [6] has proved several elegant theorems for $G(q)$, many of which are analogues of well-known properties satisfied by the Rogers-Ramanujan continued fraction

$$
F(q):=\frac{q^{1 / 5}}{1}+\frac{q}{1}+\frac{q^{2}}{1}+\frac{q^{3}}{1}+\cdots .
$$

In his first letter to Hardy, Ramanujan asserted that $F\left(e^{-\pi \sqrt{n}}\right)$ "can be exactly found if $n$ be any positive rational quantity." Although this claim is correct, and the values are algebraic, it is generally quite difficult to evaluate $F\left(e^{-\pi \sqrt{n}}\right)$ for specific values of $n$. See papers of Ramanathan [7]-[10] and Berndt and Chan [2] for specific evaluations. In contrast to this situation, we prove in Section 4 general formulas for $G\left(-e^{-\pi \sqrt{n}}\right)$ and $G\left(e^{-\pi \sqrt{n}}\right)$ that arise from the formulas connecting $G_{n}$ and $G_{9 n}$, and $g_{n}$ and $g_{9 n}$, respectively. We emphasize that no such formulas are known for $F\left(e^{-\pi \sqrt{n}}\right)$ and $F\left(-e^{-\pi \sqrt{n}}\right)$.

2. Formulas connecting $G_{n}$ and $G_{9 n}$, and $g_{n}$ and $g_{9 n}$

THEOREM 1. Let

$$
p=G_{n}^{4}+G_{n}^{-4}
$$

Then

$$
\begin{aligned}
& \text { 2) } G_{9 n}=G_{n}\left(p+\sqrt{p^{2}-1}\right)^{1 / 6} \\
& \times\left\{\sqrt{\frac{p^{2}-2+\sqrt{\left(p^{2}-1\right)\left(p^{2}-4\right)}}{2}}+\sqrt{\frac{p^{2}-4+\sqrt{\left(p^{2}-1\right)\left(p^{2}-4\right)}}{2}}\right\}^{1 / 3} .
\end{aligned}
$$

Proof. For brevity, we set $G=G_{n}$ throughout the proof.

We shall employ Entry 15(xii) in Chapter 19 of Ramanujan's second notebook [11], [1, p. 231]. Let

$$
P=\{16 \alpha \beta(1-\alpha)(1-\beta)\}^{1 / 8} \text { and } Q=\left(\frac{\beta(1-\beta)}{\alpha(1-\alpha)}\right)^{1 / 4},
$$

where $\beta$ has degree 3 . Then

$$
Q+\frac{1}{Q}+2 \sqrt{2}\left(P-\frac{1}{P}\right)=0 .
$$

Now from (1.5) and (1.7), when $q=\exp (-\pi \sqrt{n})$,

$$
G=G_{n}=\{4 \alpha(1-\alpha)\}^{-1 / 24} \quad \text { and } \quad G_{9 n}=\{4 \beta(1-\beta)\}^{-1 / 24} .
$$


Hence, by (2.3), when $q=\exp (-\pi \sqrt{n}), P=\left(G G_{9 n}\right)^{-3}$ and $Q=\left(G / G_{9 n}\right)^{6}$. Thus, from (2.4),

$$
\left(G / G_{9 n}\right)^{6}+\left(G / G_{9 n}\right)^{-6}+2 \sqrt{2}\left(\left(G G_{9 n}\right)^{-3}-\left(G G_{9 n}\right)^{3}\right)=0 .
$$

Set $x=\left(G_{9 n} / G\right)^{3}$. Thus, $(2.5)$ can be rewritten in the form

$$
x^{4}-2 \sqrt{2} G^{6} x^{3}+2 \sqrt{2} G^{-6} x+1=0 .
$$

Rearranging and using the notation (2.1), we find that we can recast (2.6) in the form

$$
\left(x^{2}-\sqrt{2} G^{6} x+p\right)^{2}=2\left(p^{2}-1\right)\left(G^{2} x-\frac{1}{\sqrt{2}}\right)^{2} .
$$

Since $x>1$,

or

$$
x^{2}-\sqrt{2} G^{6} x+p=\sqrt{2\left(p^{2}-1\right)}\left(G^{2} x-\frac{1}{\sqrt{2}}\right),
$$

$$
x^{2}-\sqrt{2} G^{2}\left(G^{4}+\sqrt{p^{2}-1}\right) x+p+\sqrt{p^{2}-1}=0 .
$$

Noting that $x>1$ and solving for $x$, we find that

$$
\begin{aligned}
x= & \frac{1}{\sqrt{2}} G^{2}\left(G^{4}+\sqrt{p^{2}-1}\right) \\
& +\frac{1}{\sqrt{2}} \sqrt{G^{4}\left(G^{8}+2 G^{4} \sqrt{p^{2}-1}+p^{2}-1\right)-2 p-2 \sqrt{p^{2}-1}} .
\end{aligned}
$$

Now,

$$
G^{4}=\frac{1}{2}\left(p+\sqrt{p^{2}-4}\right) \quad \text { and } \quad G^{8}=\frac{1}{2}\left(p^{2}-2+p \sqrt{p^{2}-4}\right) .
$$

Thus, squaring and expanding, we find that

$$
\begin{aligned}
G^{4}\left(G^{4}\right. & \left.+\sqrt{p^{2}-1}\right)^{2} \\
= & \frac{1}{2}\left(p+\sqrt{p^{2}-4}\right) \frac{1}{2}\left(p^{2}-2+p \sqrt{p^{2}-4}\right) \\
& \quad+\left(p^{2}-2+p \sqrt{p^{2}-4}\right) \sqrt{p^{2}-1}+\frac{1}{2}\left(p+\sqrt{p^{2}-4}\right)\left(p^{2}-1\right) \\
= & \left(p+\sqrt{p^{2}-1}\right)\left(p^{2}-2+\sqrt{\left(p^{2}-1\right)\left(p^{2}-4\right)}\right)
\end{aligned}
$$

and

$$
\begin{aligned}
G^{4}\left(G^{8}+\right. & \left.2 G^{4} \sqrt{p^{2}-1}+p^{2}-1\right)-2 p-2 \sqrt{p^{2}-1} \\
= & \frac{1}{2}\left(p+\sqrt{p^{2}-4}\right) \frac{1}{2}\left(p^{2}-2+p \sqrt{p^{2}-4}\right) \\
& +\left(p^{2}-2+p \sqrt{p^{2}-4}\right) \sqrt{p^{2}-1} \\
& +\frac{1}{2}\left(p+\sqrt{p^{2}-4}\right)\left(p^{2}-1\right)-2 p-2 \sqrt{p^{2}-1} \\
= & \left(p+\sqrt{p^{2}-1}\right)\left(p^{2}-4+\sqrt{\left(p^{2}-1\right)\left(p^{2}-4\right)}\right) .
\end{aligned}
$$


Using (2.9) and (2.10) in (2.7), we deduce that

$$
\begin{aligned}
x= & \frac{1}{\sqrt{2}} \sqrt{\left(p+\sqrt{p^{2}-1}\right)\left(p^{2}-2+\sqrt{\left(p^{2}-1\right)\left(p^{2}-4\right)}\right)} \\
& +\frac{1}{\sqrt{2}} \sqrt{\left(p+\sqrt{p^{2}-1}\right)\left(p^{2}-4+\sqrt{\left(p^{2}-1\right)\left(p^{2}-4\right)}\right)} .
\end{aligned}
$$

Recalling that $x=\left(G_{9 n} / G\right)^{3}$, we see that (2.11) is equivalent to (2.2), and so the proof is complete.

We next prove the aforementioned result found on page 318 in Ramanujan's first notebook.

Theorem 2. Let

$$
p=g_{n}^{4}-g_{n}^{-4} .
$$

Then

$$
\times\left\{\sqrt{\frac{p^{2}+4+\sqrt{\left(p^{2}+1\right)\left(p^{2}+4\right)}}{2}}+\sqrt{\frac{p^{2}+2+\sqrt{\left(p^{2}+1\right)\left(p^{2}+4\right)}}{2}}\right\}^{1 / 3} .
$$

Proof. Set $g=g_{n}$ throughout the proof. Using (1.7) and (1.4), we rewrite (2.5) in the form

$$
\begin{aligned}
\frac{\sqrt{q}\left(-q ; q^{2}\right)_{\infty}^{6}}{\left(-q^{3} ; q^{6}\right)_{\infty}^{6}}+ & \frac{\left(-q^{3} ; q^{6}\right)_{\infty}^{6}}{\sqrt{q}\left(-q ; q^{2}\right)_{\infty}^{6}} \\
& +\frac{8 \sqrt{q}}{\left(-q ; q^{2}\right)_{\infty}^{3}\left(-q^{3} ; q^{6}\right)_{\infty}^{3}}-\frac{\left(-q ; q^{2}\right)_{\infty}^{3}\left(-q^{3} ; q^{6}\right)_{\infty}^{3}}{\sqrt{q}}=0 .
\end{aligned}
$$

Multiplying both sides by $\sqrt{q}$ and then replacing $q$ by $-q$, we find that

$$
-\frac{q\left(q ; q^{2}\right)_{\infty}^{6}}{\left(q^{3} ; q^{6}\right)_{\infty}^{6}}+\frac{\left(q^{3} ; q^{6}\right)_{\infty}^{6}}{\left(q ; q^{2}\right)_{\infty}^{6}}-\frac{8 q}{\left(q ; q^{2}\right)_{\infty}^{3}\left(q^{3} ; q^{6}\right)_{\infty}^{3}}-\left(q ; q^{2}\right)_{\infty}^{3}\left(q^{3} ; q^{6}\right)_{\infty}^{3}=0 .
$$

Using (1.7) and (1.4) again, we find that

$$
-\left(g / g_{9 n}\right)^{6}+\left(g_{9 n} / g\right)^{6}-2 \sqrt{2}\left(g g_{9 n}\right)^{-3}-2 \sqrt{2}\left(g g_{9 n}\right)^{3}=0 .
$$

Setting $x=\left(g_{9 n} / g\right)^{3}$, we deduce that

$$
x^{4}-2 \sqrt{2} g^{6} x^{3}-2 \sqrt{2} g^{-6} x-1=0 .
$$

Recalling the notation (2.12), we see that the last equation can be rewritten in the form

$$
\left(x^{2}-\sqrt{2} g^{6} x-p\right)^{2}=2\left(p^{2}+1\right)\left(g^{2} x+\frac{1}{\sqrt{2}}\right)^{2} .
$$


It should now be clear that the remainder of the proof is completely analogous to that for Theorem 1, and so we omit the rest of the proof.

The cube roots in (2.2) and (2.13) are not very attractive, and usually in applications Ramanujan found more appealing expressions for these cube roots. Ramanujan had an amazing ability for denesting and simplifying radicals, and undoubtedly we do not have the insights into radicals that Ramanujan had. However, it seems quite likely that in several instances Ramanujan used the following elementary result from Carr's book [5, p. 52]. Since Carr does not give a proof and since he adds the extraneous hypothesis that $\left(a^{2}-b\right)^{1 / 3}$ be a perfect cube, we provide a proof here.

Lemma 3. Suppose that $c:=\left(a^{2}-b\right)^{1 / 3}$. Then we can write

$$
(a+\sqrt{b})^{1 / 3}=x+\sqrt{y},
$$

where

$$
4 x^{3}-3 c x=a
$$

and

$$
y=x^{2}-c .
$$

Pr o of. From (2.14) we easily see that

$$
\frac{(a-\sqrt{b})^{1 / 3}}{c}=\frac{x-\sqrt{y}}{x^{2}-y} .
$$

Suppose we set $c=x^{2}-y$, so that

$$
(a-\sqrt{b})^{1 / 3}=x-\sqrt{y} .
$$

Cubing both sides of (2.14) and (2.17) and solving for $a$, we find that

$$
a=x^{3}+3 x y .
$$

But since $y=x^{2}-c$, we deduce (2.15) from (2.18).

Usually, it is best to solve (2.14) by trial or inspection, for if, for example, Cardano's method is used, the value of $x$ so obtained most frequently is the cube root that we originally sought to simplify.

Since Carr's book [5] was Ramanujan's primary source of mathematics, it seems likely that Ramanujan employed Lemma 3 in simplifications. However, because most of us do not possess Ramanujan's ability to discern algebraic relationships, we describe another procedure that rests upon elementary considerations in algebraic number theory and involves less guessing.

We see from Theorems 1 and 2 that it would be advantageous to find a number $a$ such that

$$
a^{3}:=\sqrt{b+1+c \sqrt{d}}+\sqrt{b+c \sqrt{d}} .
$$


Then

$$
a^{-3}:=\sqrt{b+1+c \sqrt{d}}-\sqrt{b+c \sqrt{d}}
$$

and

$$
a^{3}+a^{-3}=2 \sqrt{b+1+c \sqrt{d}} .
$$

Since

set

$$
a^{3}+a^{-3}=\left(a+\frac{1}{a}\right)^{3}-3\left(a+\frac{1}{a}\right)
$$

$$
u=a+\frac{1}{a},
$$

so that, after squaring, (2.20) takes the shape

$$
u^{2}\left(u^{2}-3\right)^{2}=4(b+1+c \sqrt{d}) .
$$

Assuming the relevant expressions above are algebraic integers, we find that, upon taking norms in (2.22),

$$
N\left(u^{2}\right) N^{2}\left(u^{2}-3\right)=N(4(b+1+c \sqrt{d})) .
$$

Using (2.23), we determine $u$. We then solve (2.21) for $a$.

\section{Five class invariants}

THEOREM 4.

$$
G_{117}=\frac{1}{2}\left(\frac{3+\sqrt{13}}{2}\right)^{1 / 4}(2 \sqrt{3}+\sqrt{13})^{1 / 6}\left(3^{1 / 4}+\sqrt{4+\sqrt{3}}\right) .
$$

Pr o of. Let $n=13$ in Theorem 1. From Weber's treatise [23, p. 721], or Ramanujan's paper [11],

$$
G_{13}=\left(\frac{3+\sqrt{13}}{2}\right)^{1 / 4}
$$

By $(2.1), p=\sqrt{13}$. Thus, by a direct application of $(2.2)$,

$$
G_{117}=\left(\frac{3+\sqrt{13}}{2}\right)^{1 / 4}(2 \sqrt{3}+\sqrt{13})^{1 / 6}\left\{\sqrt{\frac{11+6 \sqrt{3}}{2}}+\sqrt{\frac{9+6 \sqrt{3}}{2}}\right\}^{1 / 3} .
$$

(On page 314 in his first notebook [12], Ramanujan recorded $G_{117}$ in the form given above, which is strong evidence that Ramanujan utilized Theorem 1 as we have done.) It therefore remains to show that

$$
\left\{\sqrt{\frac{11+6 \sqrt{3}}{2}}+\sqrt{\frac{9+6 \sqrt{3}}{2}}\right\}^{1 / 3}=\frac{1}{2}\left(3^{1 / 4}+\sqrt{4+\sqrt{3}}\right) .
$$


We apply Lemma 3 with

$$
a=\sqrt{\frac{11+6 \sqrt{3}}{2}} \text { and } b=\frac{9+6 \sqrt{3}}{2} .
$$

Thus, $c=1$. We therefore need to solve

$$
4 x^{3}-3 x=\sqrt{\frac{11+6 \sqrt{3}}{2}} .
$$

To solve this by inspection, it perhaps is best to square both sides and set $x=t^{2}$. Thus,

$$
t(4 t-3)^{2}=\frac{11+6 \sqrt{3}}{2}
$$

It is not difficult to see that $t=1+\sqrt{3} / 4$. Hence, $x=\frac{1}{2} \sqrt{4+\sqrt{3}}$, and, from (2.16), $y=\sqrt{3} / 4$. Thus, (3.2) follows, and the proof is complete.

Alternatively, in the notation (2.19) and (2.21), by (2.22), we want to solve

$$
u^{2}\left(u^{2}-3\right)^{2}=22+12 \sqrt{3}
$$

Factoring in $Z[\sqrt{3}]$, we deduce from (2.23) that

$$
N\left(u^{2}\right) N^{2}\left(u^{2}-3\right)=N(22+12 \sqrt{3})=2^{2} \cdot 13 .
$$

Then

$$
\pm 2=N\left(u^{2}-3\right)=: N(A+B \sqrt{3})=A^{2}-3 B^{2} .
$$

Choose $A=1=B$. We now observe that

$$
N\left(u^{2}\right)=N(4+\sqrt{3})=13
$$

as required. It is easily checked that, when $u=\sqrt{4+\sqrt{3}},(3.3)$ holds. We then readily find that

$$
a=\frac{1}{2}\left(3^{1 / 4}+\sqrt{4+\sqrt{3}}\right) .
$$

Thus, (3.2) has been shown once again.

Theorem 5.

$$
\begin{aligned}
G_{153}=\left(\sqrt{\frac{5+\sqrt{17}}{8}}+\sqrt{\frac{\sqrt{17}-3}{8}}\right)^{2} & \\
& \times\left(\sqrt{\frac{37+9 \sqrt{17}}{4}}+\sqrt{\frac{33+9 \sqrt{17}}{4}}\right)^{1 / 3} .
\end{aligned}
$$

Proof. Set $n=17$ in Theorem 1. By Weber's treatise [23, p. 721], or Ramanujan's paper [11],

$$
G_{17}=\sqrt{\frac{5+\sqrt{17}}{8}}+\sqrt{\frac{\sqrt{17}-3}{8}} .
$$


Since

$$
G_{17}^{4}=\frac{5+\sqrt{17}}{4}+\frac{(1+\sqrt{17})^{3 / 2}}{4 \sqrt{2}},
$$

from (2.1) we find that

$$
p=\frac{5+\sqrt{17}}{2} .
$$

Also,

$$
p+\sqrt{p^{2}-1}=\frac{5+\sqrt{17}}{2}+\sqrt{\frac{19+5 \sqrt{17}}{2}} .
$$

Thus, from Theorem 1 ,

$$
\begin{aligned}
G_{153}= & \left(\sqrt{\frac{5+\sqrt{17}}{8}}+\sqrt{\frac{\sqrt{17}-3}{8}}\right)\left(\frac{5+\sqrt{17}}{2}+\sqrt{\frac{19+5 \sqrt{17}}{2}}\right)^{1 / 6} \\
& \times\left\{\sqrt{\frac{17+5 \sqrt{17}+\sqrt{(19+5 \sqrt{17})(13+5 \sqrt{17})}}{4}}\right. \\
& \left.+\sqrt{\frac{13+5 \sqrt{17}+\sqrt{(19+5 \sqrt{17})(13+5 \sqrt{17})}}{4}}\right\}^{1 / 3} .
\end{aligned}
$$

However, note that

$$
(19+5 \sqrt{17})(13+5 \sqrt{17})=672+160 \sqrt{17}=(20+4 \sqrt{17})^{2} .
$$

Hence,

$$
\begin{aligned}
G_{153}= & \left(\sqrt{\frac{5+\sqrt{17}}{8}}+\sqrt{\frac{\sqrt{17}-3}{8}}\right)\left(\frac{5+\sqrt{17}}{2}+\sqrt{\frac{19+5 \sqrt{17}}{2}}\right)^{1 / 6} \\
& \times\left\{\sqrt{\frac{37+9 \sqrt{17}}{4}}+\sqrt{\frac{33+9 \sqrt{17}}{4}}\right\}^{1 / 3} .
\end{aligned}
$$

Comparing the equality above with (3.4), by (3.5), we see that it remains to show that

$$
\frac{5+\sqrt{17}}{2}+\sqrt{\frac{19+5 \sqrt{17}}{2}}=G_{17}^{6},
$$

which is rather curious indeed. Note that

$$
\left(\sqrt{\frac{7+\sqrt{17}}{4}}+\sqrt{\frac{3+\sqrt{17}}{4}}\right)^{2}=\frac{5+\sqrt{17}}{2}+\sqrt{\frac{19+5 \sqrt{17}}{2}} .
$$


Thus, by (3.6), it suffices to show that

$$
\sqrt{\frac{7+\sqrt{17}}{4}}+\sqrt{\frac{3+\sqrt{17}}{4}}=G_{17}^{3} .
$$

In the notation (2.19) and (2.21), we solve

$$
u^{2}\left(u^{2}-3\right)^{2}=7+\sqrt{17}
$$

Factoring in $Z\left[\frac{1+\sqrt{17}}{2}\right]$, we deduce that

$$
N\left(u^{2}\right) N^{2}\left(u^{2}-3\right)=N(7+\sqrt{17})=2^{5} .
$$

We attempt to solve

$$
\pm 4=N\left(u^{2}-3\right)=: N\left(A+B \frac{1+\sqrt{17}}{2}\right)=A^{2}+A B-4 B^{2} .
$$

Take $A=-1$ and $B=1$. Then $u^{2}=(5+\sqrt{17}) / 2$ and $N\left(u^{2}\right)=2$. A simple calculation shows that (3.8) indeed holds. Lastly, we find that

$$
a=\sqrt{\frac{5+\sqrt{17}}{8}}+\sqrt{\frac{\sqrt{17}-3}{8}} .
$$

By (3.5), we conclude that (3.7) holds to complete the proof.

\section{THeOrem 6.}

(3.9) $\quad G_{441}$

$$
=\sqrt{\frac{2+\sqrt{7}+\sqrt{7+4 \sqrt{7}}}{2}} \sqrt{\frac{\sqrt{3}+\sqrt{7}}{2}}(2+\sqrt{3})^{1 / 6} \sqrt{\frac{\sqrt{3+\sqrt{7}}+6^{1 / 4} 7^{1 / 8}}{\sqrt{3+\sqrt{7}}-6^{1 / 4} 7^{1 / 8}}} .
$$

Proof. We apply Theorem 1 with $n=49$. From Weber's treatise [23, p. 723], or Ramanujan's paper [11],

$$
G_{49}=\frac{\sqrt{4+\sqrt{7}}+7^{1 / 4}}{2} .
$$

It is easily checked that

$$
\frac{\sqrt{4+\sqrt{7}}+7^{1 / 4}}{2}=\sqrt{\frac{2+\sqrt{7}+\sqrt{7+4 \sqrt{7}}}{2}} .
$$

After a somewhat lengthy calculation, we find that

$$
G_{49}^{ \pm 4}=\frac{9+4 \sqrt{7}}{2} \pm \frac{(9+3 \sqrt{7}) 7^{1 / 4}}{2 \sqrt{2}} .
$$

Thus, $p=9+4 \sqrt{7}$. After a mild calculation, 


$$
\begin{aligned}
p+\sqrt{p^{2}-1} & =9+4 \sqrt{7}+2 \sqrt{3} \sqrt{16+6 \sqrt{7}} \\
& =9+4 \sqrt{7}+2 \sqrt{3}(3+\sqrt{7}) \\
& =(2+\sqrt{3})(3 \sqrt{3}+2 \sqrt{7}) \\
& =(2+\sqrt{3})\left(\frac{\sqrt{3}+\sqrt{7}}{2}\right)^{3} .
\end{aligned}
$$

Using (3.10)-(3.12) in Theorem 1, we deduce that

$$
\begin{aligned}
G_{441}= & \sqrt{\frac{2+\sqrt{7}+\sqrt{7+4 \sqrt{7}}}{2}} \sqrt{\frac{\sqrt{3}+\sqrt{7}}{2}}(2+\sqrt{3})^{1 / 6} \\
& \times\left\{\sqrt{\frac{191+72 \sqrt{7}+A}{2}}+\sqrt{\frac{189+72 \sqrt{7}+A}{2}}\right\}^{1 / 3},
\end{aligned}
$$

where

$$
\begin{aligned}
A & :=\sqrt{(192+72 \sqrt{7})(189+72 \sqrt{7})} \\
& =6 \sqrt{2016+762 \sqrt{7}}=6 \cdot 7^{1 / 4} \sqrt{3 / 2}(3+\sqrt{7})^{2} .
\end{aligned}
$$

Using this calculation in (3.13), we see from (3.13) and (3.9) that it remains to prove that

$$
\begin{aligned}
\left\{\sqrt{\frac{191+72 \sqrt{7}+6 \cdot 7^{1 / 4} \sqrt{3 / 2}(3+\sqrt{7})^{2}}{2}}\right. & \\
& \left.+\sqrt{\frac{189+72 \sqrt{7}+6 \cdot 7^{1 / 4} \sqrt{3 / 2}(3+\sqrt{7})^{2}}{2}}\right\}^{1 / 3} \\
= & \sqrt{\frac{\sqrt{3+\sqrt{7}}+6^{1 / 4} 7^{1 / 8}}{\sqrt{3+\sqrt{7}}-6^{1 / 4} 7^{1 / 8}}} \\
= & \frac{\left(\sqrt{3+\sqrt{7}}+6^{1 / 4} 7^{1 / 8}\right) \sqrt{3+\sqrt{7}+6^{1 / 2} 7^{1 / 4}}}{4} \\
= & \frac{1}{4} \sqrt{16+6 \sqrt{7}+(3+\sqrt{7}) 6^{1 / 2} 7^{1 / 4}} \\
& +\frac{1}{4} \sqrt{6 \sqrt{7}+(3+\sqrt{7}) 6^{1 / 2} 7^{1 / 4}} .
\end{aligned}
$$

We apply Lemma 3 with

$$
a=\sqrt{\frac{191+72 \sqrt{7}+6 \cdot 7^{1 / 4} \sqrt{3 / 2}(3+\sqrt{7})^{2}}{2}}
$$


and

$$
b=\frac{189+72 \sqrt{7}+6 \cdot 7^{1 / 4} \sqrt{3 / 2}(3+\sqrt{7})^{2}}{2} .
$$

Again, $c=1$. Setting $x^{2}=t$ in (2.15), we see that we must solve

$$
\begin{aligned}
t(4 t-3)^{2} & =\frac{191+72 \sqrt{7}+6 \cdot 7^{1 / 4} \sqrt{3 / 2}(3+\sqrt{7})^{2}}{2} \\
& =\frac{(16+3 \sqrt{7})(3+\sqrt{7})^{2}+6 \sqrt{6} \cdot 7^{1 / 4}(3+\sqrt{7})^{2}}{4} \\
& =\frac{(3+\sqrt{7})^{2}}{4}\left(16+3 \sqrt{7}+6 \sqrt{6} \cdot 7^{1 / 4}\right) .
\end{aligned}
$$

We now verify that

$$
t=\frac{3+\sqrt{7}}{16}\left(3+\sqrt{7}+6^{1 / 2} 7^{1 / 4}\right)
$$

Thus,

$$
x=\frac{\sqrt{(3+\sqrt{7})^{2}+(3+\sqrt{7}) 6^{1 / 2} 7^{1 / 4}}}{4}
$$

and

$$
y=6 \sqrt{7}+(3+\sqrt{7}) 6^{1 / 2} 7^{1 / 4}
$$

by (2.16). Thus, (3.14) follows, and the proof is complete.

\section{THEOREM 7.}

$$
g_{90}=(2+\sqrt{5})^{1 / 6}(\sqrt{5}+\sqrt{6})^{1 / 6}\left(\sqrt{\frac{3+\sqrt{6}}{4}}+\sqrt{\frac{\sqrt{6}-1}{4}}\right) .
$$

Proof. Set $n=10$ in Theorem 2. Now from Weber's book [23, p. 721], or Ramanujan's paper [11],

$$
g_{10}=\sqrt{\frac{1+\sqrt{5}}{2}} .
$$

An easy calculation shows that $p=\sqrt{5}$. Note also that

$$
g_{10}=\sqrt{\frac{1+\sqrt{5}}{2}}=\left(\left(\frac{1+\sqrt{5}}{2}\right)^{3}\right)^{1 / 6}=(2+\sqrt{5})^{1 / 6} .
$$

Thus, from (2.13) and (3.16),

$$
g_{90}=(2+\sqrt{5})^{1 / 6}(\sqrt{5}+\sqrt{6})^{1 / 6}\left\{\sqrt{\frac{9+3 \sqrt{6}}{2}}+\sqrt{\frac{7+3 \sqrt{6}}{2}}\right\}^{1 / 3} .
$$


Comparing (3.17) and (3.15), we see that we must prove that

$$
\left\{\sqrt{\frac{9+3 \sqrt{6}}{2}}+\sqrt{\frac{7+3 \sqrt{6}}{2}}\right\}^{1 / 3}=\sqrt{\frac{3+\sqrt{6}}{4}}+\sqrt{\frac{\sqrt{6}-1}{4}} .
$$

In the notation (2.19) and (2.21), we solve

$$
u^{2}\left(u^{2}-3\right)^{2}=18+6 \sqrt{6} \text {. }
$$

Factoring in $\mathbb{Z}[\sqrt{6}]$, we find that

$$
N\left(u^{2}\right) N^{2}\left(u^{2}-3\right)=N(18+6 \sqrt{6})=3 \cdot 6^{2} .
$$

We thus want to solve

$$
\pm 6=N\left(u^{2}-3\right)=: N(A+B \sqrt{6})=A^{2}-6 B^{2} .
$$

Choose $A=0$ and $B=1$, so that $u^{2}=3+\sqrt{6}$ and $N\left(u^{2}\right)=3$. It is trivial to see that (3.19) is satisfied. Then

$$
a=\sqrt{\frac{3+\sqrt{6}}{4}}+\sqrt{\frac{\sqrt{6}-1}{4}},
$$

and the verification of (3.18) is complete.

\section{THEOREM 8.}

$$
g_{198}=\sqrt{1+\sqrt{2}}(4 \sqrt{2}+\sqrt{33})^{1 / 6}\left(\sqrt{\frac{9+\sqrt{33}}{8}}+\sqrt{\frac{1+\sqrt{33}}{8}}\right) .
$$

Proof. Set $n=22$ in Theorem 2. From Weber's work [23, p. 722], or Ramanujan's paper [11],

$$
g_{22}=\sqrt{1+\sqrt{2}} .
$$

An easy calculation yields $p=4 \sqrt{2}$. Thus, from Theorem 2 ,

(3.22) $g_{198}=\sqrt{1+\sqrt{2}}(4 \sqrt{2}+\sqrt{33})^{1 / 6}\{\sqrt{18+3 \sqrt{33}}+\sqrt{17+3 \sqrt{33}}\}^{1 / 3}$.

By (3.20) and (3.22), we must show that

$$
\{\sqrt{18+3 \sqrt{33}}+\sqrt{17+3 \sqrt{33}}\}^{1 / 3}=\sqrt{\frac{9+\sqrt{33}}{8}}+\sqrt{\frac{1+\sqrt{33}}{8}} .
$$

In the notation of (2.19) and (2.21), we seek to solve

$$
u^{2}\left(u^{2}-3\right)^{2}=4(18+3 \sqrt{33}) \text {. }
$$

Thus,

$$
N\left(u^{2}\right) N^{2}\left(u^{2}-3\right)=N(4(18+3 \sqrt{33}))=2^{4} \cdot 3^{3} .
$$

Factoring in $\mathbb{Z}\left[\frac{1+\sqrt{33}}{2}\right]$, we attempt to find a solution of

$$
\pm 6=N\left(u^{2}-3\right)=: N\left(A+B \frac{1+\sqrt{33}}{2}\right)=A^{2}+A B-8 B^{2} .
$$


Let $A=1=B$, so that $u^{2}=(9+\sqrt{33}) / 2$ and $N\left(u^{2}\right)=12$. It is easily checked that (3.24) holds. Hence,

$$
a=\sqrt{\frac{9+\sqrt{33}}{8}}+\sqrt{\frac{1+\sqrt{33}}{8}} .
$$

Thus, we can deduce (3.23), and the proof is complete.

\section{Explicit evaluations of Ramanujan's cubic continued fraction}

Theorem 9. Let $G(q)$ be defined by (1.8) and $p$ be defined by (2.1). Then

$$
\begin{aligned}
& G\left(-e^{-\pi \sqrt{n}}\right)=-\sqrt{\frac{p-\sqrt{p^{2}-1}}{p+\sqrt{p^{2}-4}}} \\
& \quad \times\left(\sqrt{\frac{p^{2}-2+\sqrt{\left(p^{2}-1\right)\left(p^{2}-4\right)}}{2}}-\sqrt{\frac{p^{2}-4+\sqrt{\left(p^{2}-1\right)\left(p^{2}-4\right)}}{2}}\right) .
\end{aligned}
$$

Proof. It was first proved by Watson [15] that

$$
G(q)=q^{1 / 3} \frac{\chi(-q)}{\chi^{3}\left(-q^{3}\right)},
$$

where $\chi(q)$ is defined by (1.4). (For references to other proofs of (4.1), see $\left[1\right.$, pp. 346, 347].) For $q=-e^{-\pi \sqrt{n}}$, we have, by (4.1) and (1.7),

$$
\begin{aligned}
G\left(-e^{-\pi \sqrt{n}}\right) & =-e^{-\pi \sqrt{n} / 3} \frac{\chi\left(e^{-\pi \sqrt{n}}\right)}{\chi^{3}\left(e^{-3 \pi \sqrt{n}}\right)} \\
& =-\frac{1}{\sqrt{2}} \cdot \frac{G_{n}}{G_{9 n}^{3}}=-\frac{1}{\sqrt{2}} G_{n}^{-2}\left(\frac{G_{n}}{G_{9 n}}\right)^{3} .
\end{aligned}
$$

If

$$
u(p):=\sqrt{\frac{p^{2}-4+\sqrt{\left(p^{2}-1\right)\left(p^{2}-4\right)}}{2}},
$$

from (2.2) and (4.2), we find that

$$
G\left(-e^{-\pi \sqrt{n}}\right)=-\frac{1}{\sqrt{2}} G_{n}^{-2} \sqrt{p-\sqrt{p^{2}-1}}(\sqrt{u(p)+1}-\sqrt{u(p)}) .
$$

But, from (2.8),

$$
G_{n}^{-2}=\left(\frac{p+\sqrt{p^{2}-4}}{2}\right)^{-1 / 2}
$$

Substituting (4.4) into (4.3), we complete the proof.

We next illustrate Theorem 9 with a few examples. 
EXAMPLE 1.

$$
G\left(-e^{-\pi}\right)=\frac{1-\sqrt{3}}{2} .
$$

Pr o of. Let $n=1$. Then, trivially, $G_{1}=1$ and $p=2$. Thus, by Theorem 9 ,

$$
G\left(-e^{-\pi}\right)=-\left(\frac{2-\sqrt{3}}{2}\right)^{1 / 2}=\frac{1-\sqrt{3}}{2} .
$$

See $[6]$ for another proof.

EXAMPLE 2.

$$
G\left(-e^{-\pi \sqrt{5}}\right)=\frac{(\sqrt{5}-3)(\sqrt{5}-\sqrt{3})}{4} .
$$

Pr o of. From Weber's treatise [23, p. 721] or Ramanujan's paper [11],

$$
G_{5}=\left(\frac{\sqrt{5}+1}{2}\right)^{1 / 4}
$$

It easily follows that $G_{5}^{4}+G_{5}^{-4}=\sqrt{5}$, and so

$$
G\left(-e^{\pi \sqrt{5}}\right)=-\sqrt{\frac{\sqrt{5}-2}{\sqrt{5}+1}}\left(\sqrt{\frac{5}{2}}-\sqrt{\frac{3}{2}}\right)=\frac{(\sqrt{5}-3)(\sqrt{5}-\sqrt{3})}{4} .
$$

Similarly, using the value of $G_{13}$, given at the beginning of the proof of Theorem 4 , and the value $G_{37}=(\sqrt{37}+6)^{1 / 4}([23$, p. 722$]$, [11]), we can deduce the following evaluations.

EXAMPLE 3.

$$
G\left(-e^{-\pi \sqrt{13}}\right)=-\sqrt{\frac{\sqrt{13}-2 \sqrt{3}}{\sqrt{13}+3}}\left(\sqrt{\frac{11+6 \sqrt{3}}{2}}-\sqrt{\frac{9+6 \sqrt{3}}{2}}\right) .
$$

EXAMPLE 4.

$$
G\left(-e^{-\pi \sqrt{37}}\right)=-\sqrt{\frac{2 \sqrt{37}-7 \sqrt{3}}{2 \sqrt{37}+12}}\left(\sqrt{\frac{146+84 \sqrt{3}}{2}}-\sqrt{\frac{144+84 \sqrt{3}}{2}}\right) .
$$

Then

Theorem 10. Let $G(q)$ be defined by (1.8) and $p$ be defined by (2.12).

$$
\begin{array}{r}
G\left(e^{-\pi \sqrt{n}}\right)=\sqrt{\frac{\sqrt{p^{2}+1}-p}{\sqrt{p^{2}+4}+p}}\left(\sqrt{\frac{p^{2}+4+\sqrt{\left(p^{2}+1\right)\left(p^{2}+4\right)}}{2}}\right. \\
\left.-\sqrt{\frac{p^{2}+2+\sqrt{\left(p^{2}+1\right)\left(p^{2}+4\right)}}{2}}\right) .
\end{array}
$$


Proof. Arguing as in the preceding proof, we deduce from (4.1) and (1.7) that

$$
G\left(e^{-\pi \sqrt{n}}\right)=\frac{1}{\sqrt{2}} \cdot \frac{g_{n}}{g_{9 n}^{3}}=\frac{1}{\sqrt{2}} g_{n}^{-2}\left(\frac{g_{n}}{g_{9 n}}\right)^{3} .
$$

If

$$
v(p):=\sqrt{\frac{p^{2}+2+\sqrt{\left(p^{2}+1\right)\left(p^{2}+4\right)}}{2}},
$$

then, by (4.5) and (2.13),

$$
G\left(e^{-\pi \sqrt{n}}\right)=\frac{1}{\sqrt{2}} g_{n}^{-2} \sqrt{\sqrt{p^{2}+1}-p}(\sqrt{v(p)+1}-\sqrt{v(p)}) .
$$

However, from (2.12), since $p>0$,

$$
g_{n}^{-2}=\left(\frac{\sqrt{p^{2}+4}+p}{2}\right)^{-1 / 2} .
$$

Putting (4.7) in (4.6), we complete the proof.

We give a few examples to illustrate Theorem 10.

EXAMPLE 5.

$$
G\left(e^{-\pi \sqrt{2}}\right)=\frac{\sqrt{6}-2}{2} .
$$

Proof. Let $n=2$. Then ([23, p. 721], [11]) $g_{2}=1$. Hence, $p=0$, and by Theorem 10,

$$
G\left(e^{-\pi \sqrt{2}}\right)=\frac{1}{\sqrt{2}}\left(\sqrt{\frac{4+2}{2}}-\sqrt{\frac{2+2}{2}}\right)=\frac{\sqrt{6}-2}{2} .
$$

See also [6] for this evaluation.

Using $(3.16),(3.21)$, and the value $g_{58}=\sqrt{(\sqrt{29}+5) / 2}$, we can also deduce the following values.

EXAMPLE 6.

$$
G\left(e^{-\pi \sqrt{10}}\right)=\frac{\sqrt{9+3 \sqrt{6}}-\sqrt{7+3 \sqrt{6}}}{(1+\sqrt{5}) \sqrt{\sqrt{6}+\sqrt{5}}} .
$$

EXAMPLE 7.

$$
G\left(e^{-\pi \sqrt{22}}\right)=\frac{\sqrt{36+6 \sqrt{33}}-\sqrt{34+6 \sqrt{33}}}{(2+2 \sqrt{2}) \sqrt{\sqrt{33}+\sqrt{32}}} .
$$


EXAMPLE 8.

$$
G\left(e^{-\pi \sqrt{58}}\right)=\frac{\sqrt{729+297 \sqrt{6}}-\sqrt{727+297 \sqrt{6}}}{(5+\sqrt{29}) \sqrt{11 \sqrt{6}+5 \sqrt{29}}} .
$$

Example 6 appears in Ramanujan's lost notebook [14, p. 366] and was first proved by K. G. Ramanathan [7].

We give our thanks to Doug Bowman for informing us of the version of Lemma 3 in Carr's book [5].

\section{References}

[1] B. C. Berndt, Ramanujan's Notebooks, Part III, Springer, New York, 1994.

[2] B. C. Berndt and H. H. Chan, Some values for the Rogers-Ramanujan continued fraction, Canad. J. Math. 20 (1995).

[3] B. C. Berndt, H. H. Chan and L.-C. Zhang, Ramanujan's class invariants, Kronecker's limit formula, and modular equations, to appear.

[4] J. M. and P. B. Borwein, Pi and the AGM, Wiley, New York, 1987.

[5] G. S. Carr, Formulas and Theorems in Pure Mathematics, 2nd ed., Chelsea, New York, 1970.

[6] H. H. Chan, On Ramanujan's cubic continued fraction, Acta Arith., to appear.

[7] K. G. Ramanathan, On Ramanujan's continued fraction, ibid. 43 (1984), 209-226.

[8] -, On the Rogers-Ramanujan continued fraction, Proc. Indian Acad. Sci. Math. Sci. 93 (1984), 67-77.

[9] —, Ramanujan's continued fraction, Indian J. Pure Appl. Math. 16 (1985), 695-724.

[10] —, Some applications of Kronecker's limit formula, J. Indian Math. Soc. 52 (1987), 71-89.

[11] S. Ramanujan, Modular equations and approximations to $\pi$, Quart. J. Math. (Oxford) 45 (1914), 350-372.

[12] - Notebooks (2 volumes), Tata Institute of Fundamental Research, Bombay, 1957.

[13] —, Collected Papers, Chelsea, New York, 1962.

[14] - , The Lost Notebook and Other Unpublished Papers, Narosa, New Delhi, 1988.

[15] G. N. Watson, Theorems stated by Ramanujan (IX): two continued fractions, J. London Math. Soc. 4 (1929), 231-237.

[16] - Theorems stated by Ramanujan (XIV): a singular modulus, ibid. 6 (1931), 126132 .

[17] —, Some singular moduli (I), Quart. J. Math. 3 (1932), 81-98.

[18] —, Some singular moduli (II), ibid., 189-212.

[19] —, Singular moduli (3), Proc. London Math. Soc. 40 (1936), 83-142.

[20] - Singular moduli (4), Acta Arith. 1 (1936), 284-323.

[21] —, Singular moduli (5), Proc. London Math. Soc. 42 (1937), 377-397.

[22] —, Singular moduli (6), ibid., 398-409. 
[23] H. Weber, Lehrbuch der Algebra, dritter Band, Chelsea, New York, 1961.

DEPARTMENT OF MATHEMATICS

UNIVERSITY OF ILLINOIS

1409 WEST GREEN STREET

URBANA, ILLINOIS 61801

U.S.A.

SCHOOL OF MATHEMATICS

INSTITUTE FOR ADVANCED STUDY

PRINCETON, NEW JERSEY 08540

U.S.A.
DEPARTMENT OF MATHEMATICS SOUTHWEST MISSOURI STATE UNIVERSITY SPRINGFIELD, MISSOURI 65804

Received on 8.1.1995

and in revised form on 13.2.1995 